This information is current as of April 26, 2023.

\title{
The Uncertainty of Science and the Science of Uncertainty
}

M. Castillo

AJNR Am J Neuroradiol 2010, 31 (10) 1767-1768

doi: https://doi.org/10.3174/ajnr.A2106

http://www.ajnr.org/content/31/10/1767 


\section{PERSPECTIVES}

\section{The Uncertainty of Science and the Science of Uncertainty}

$\mathbf{H}^{2}$ alfway through Roberto Bolaño's chef d'oeuvre 2666: A Novel (Farrar, Straus and Giroux, 2004), one character declares, "If you know, you know, if you do not know, you learn." In that book, 4 academics, a journalist, and several policemen search for the bizarre truth behind a horrific series of murders. Of course, like in many of Bolano's books, the ending remains open and the truth elusive, and it seems to say, life is uncertain. If life is uncertain, so must be all activities it encompasses, including science. The famous physicist Richard Feynman said that all scientific knowledge is uncertain and uncertainty is a very important part of it. If you think you got something right, if you already made up your mind, you may not consider other options and will be unable to truly solve the problem. ${ }^{1}$ He goes on to say that if we are free of doubt and ignorance, we will not get any new ideas and make no progress. Others hold a similar point of view, supporting that uncertainty is one of the most important defining features of science. $^{2}$

All interpretations and reports issued by radiologists contain a degree of uncertainty. Indeed, because medicine as a whole is an uncertain science, they must reflect it. ${ }^{3}$ Uncertainty in radiology reports has been studied. An investigation assessed the 18 most common expressions of probability in a series of imaging studies reports. ${ }^{4}$ The authors asked 11 individuals to rate their interpretation of expressions used by radiologists to define probability and uncertainty. Expressions with a high probability of consistency were "absent," "excludes," "certain," and "definite." On the basis of their results, the authors suggested expressions such as "no evidence of," "unable to exclude," "appears present," and "appears absent" rated low in consistency and should be avoided. This is important because these are some of the expressions most used when "hedging" and may lead to a misunderstanding of our reports. Radiologists must learn how to manage uncertainty and avoid, as much as possible, using terms that reflect it, to prevent it from spreading to our clinical colleagues and referring physicians.

Because we radiologists face uncertainty in many studies we interpret, a different article looked at our reactions when unexpected findings were present on screening studies. ${ }^{5}$ These investigators studied radiologists' reactions to uncertainty and practice factors associated with them. Terms used to describe these reactions included "stress," "uneasy," "troubles me," "anxious," and "concerned." Three factors were directly linked to these reactions and included sex, interpretative volume, and years in practice. As expected, more experienced and older radiologists were less affected by uncertainty, but unexpectedly, women had less intense reactions toward it than men. Past involvement in medicolegal issues did not increase anxiety and uncertainty. Another important observation made by the authors is that radiologists experience more intense reactions than those reported in other medical specialties. I think this is something for us all to keep in mind when residents begin their radiology training and are exposed to great degrees of uncertainty encountered in the vast field of imaging. Uncertainty is linked to ambiguity and conspicuity. Less ambiguous and more conspicuous findings result in less uncertainty. More ambiguity and less conspicuity lead to greater numbers of false-positive results. In another study, detection probability and certainty had a direct relationship with lesion conspicuity.

If our ability to detect abnormalities is based mainly on experience (volume or work previously done and age), it is empiric by definition. Knowledge of theory and training should help lessen this empiric nature. Logical arguments about what we see in our images lead us to make inferences. ${ }^{7}$ Inferences can be of 2 types: deductive and inductive. A deductive inference states that if the premise is true, the conclusion must also be true. This type of reasoning is commonly used in scientific prospective studies when the conclusions confirm or negate the validity of the initial hypothesis. This concept can be extended to the everyday practice of radiology; we make inferences about patients' diseases on the basis of imaging studies and later we confirm or negate these on the basis of histology, laboratory results, and/or outcome. If the conclusions disprove our initial observations, we have the tendency to go back, relook at the findings, and many times doubt their initial validity and significance. This is more common when the degree of initial uncertainty was high. Absence of ambiguity and uncertainty makes confirmation of inferences unnecessary (let's say diagnosing an epidural hematoma). Thus, when findings are pathognomonic, we are no longer "interpreting" the studies but simply stating obvious observations. Making a diagnosis based on the "Aunt Minnie" behavior (pattern recognition) is a type of deductive activity.

Any argument that is not deductive tends to be inductive, that is, its conclusion cannot be established with certainty. Inductions make our lives easier and more livable and are a critical part of human optimism. In radiology, induction is less commonly used than deduction. Those who study philosophy and logic cite a third mechanism that can help interpret our imaging studies-abduction. Abductions are essential in reaching a diagnosis and are simple guesses about what things are based on previous observations. To reach correct abductions, one must have the appropriate scientific background. Abductions also help us to organize new observations achieved by using the scientific method.

The ultimate result of radiologic inferences is a report's conclusion. The conclusion is the actual interpretation of the observations listed in the findings section of the report. Data accumulated may be analyzed by using a "frequentist" (or definitive) paradigm. Once a hypothesis is proved, it becomes a theory. In frequentism, scientific realism, and determinism, a theory is accepted as true. If the conclusion in 1 report is accepted as true, knowing it when reassessing a study may affect the subsequent interpretation. Reassessing a study without knowledge of its prior results leads us to be able to directly compare dichotomous results from 2 independent readers (ourself and the previous one), and should the results be similar, it produces a more valuable interpretation of the findings. Frequentism (a probabilistic paradigm) asks questions regarding the probability of observing the data (such as $P$ and confidence intervals in statistics). An opposing point of view was 
introduced by Bayes. ${ }^{8}$ Contrary to determinism, the Bayesian paradigm states that just because a theory agrees with our observation, it cannot be assumed to be true. Although the mathematics of frequentism are relatively simple, Bayesian ones are very complex and could not be completely understood until we had computers capable of handling incredible amounts of data.

Bayesian principles are commonly used in radiology research and depend on a priori knowledge of the performance of a test that will be administered (the predictive value of that test). We radiologists love a variation of the Bayesian paradigm that emulates our clinical practice and leaves room for uncertain conclusions - that is, we can use uncertainty to our advantage. By briefly stating the probability of our report being correct or incorrect, we never have to be absolutely right or wrong. Also, we know beforehand which imaging studies give certain answers, and thus we can efficiently use them. All of this may be used to lessen the anxiety introduced by uncertainty. The possibility of uncertainty also elicits reactions from the general public. Uncertainty can be manufactured with the idea of creating doubt in science (and other human activities).

The noxious quality of tobacco products and evidence of aspirin in the development of Reye syndrome are typical examples of situations in which interested individuals have attacked the results of science to manipulate special interests. These interest groups generally use derogatory terms such as "junk science" to derail public health regulations. Junk science is characterized by spurious inference, something that does not happen when the scientific method is rigorously followed. Unfortunately, our government has tools in place that help introduce uncertainty in the public arena. The Data Quality Act allows anyone to ask for corrections if he or she believes the data presented are not of sufficient quality, objectivity, utility, and integrity. ${ }^{9}$ Although at first this seems laudable and honest, it has been widely publicized that this provision was inserted at the request of an individual who advocated industry-funded regulatory reforms. As we must strive to limit the amount of uncertainty flowing into society, we must learn to use it on an individual basis to help us improve the practice of radiology.

During the Coen brothers' movie A Serious Man, physics professor Larry Gopnik fills an enormous chalkboard with mathematic equations and upon finishing states: "The uncertainty principle. It proves that we can't ever really know... what is going on. So it shouldn't bother you. Not being able to figure anything out. Although you will be responsible for this on the midterm." ${ }^{10}$ I like to think we as radiologists have our midterm examinations every day when we interpret cases; we are responsible for the contents of our reports even if we are uncertain as to what the images show. The enormous equation on that blackboard may actually be interpreted as representing life, at the end uncertain, and many times incomprehensible. We will never be able to figure out what is going on with every image we look at, but we are still responsible for communicating this uncertainty in the best and least ambiguous way possible to others.

\section{References}

1. The Meaning of It All. http://www.inf.fu-berlin.de/lehre/pmo/eng/FeynmanUncertainty.pdf. Accessed February 18, 2010

2. Fox RC. Medical uncertainty: an interview with Renee Fox. Second Opin 1987;6:90-105

3. Gunderman RB. Education and the art of uncertainty. Radiology 2005;237:801-02

4. Hobby JL, Tom BDM, Todd C, et al. Communication of doubt and certainty in radiological reports. Br J Radiol 2000;73:999-1001

5. Careny PA, Elmore JG, Abraham LA, et al. Radiologist uncertainty and interpretation of screening. Med Decis Making 2004;24:255-64

6. Revesz G. Conspicuity and uncertainty in the radiographic detection of lesions. Radiology 1985;154:625-28

7. Sistrom C. Interference and uncertainly in radiology. Acad Radiol 2006; $13: 580-88$

8. Bayes T. An essay toward solving a problem in the doctrine of chances. Philosophical Transactions of the Royal Society of London 1764;53:370-418

9. Michaels D, Monforton C. Manufacturing uncertainly: contested science and the protection of the public's health and environment. Am J Public Health 2005;95:S39-48

10. Coen J, Coen E. A Serious Man. http://www.coenbrothers.net/scripts/aseriousman.pdf. Accessed February 23, 2010

M. Castillo

Editor-in-Chief

DOI 10.3174/ajnr.A2106 